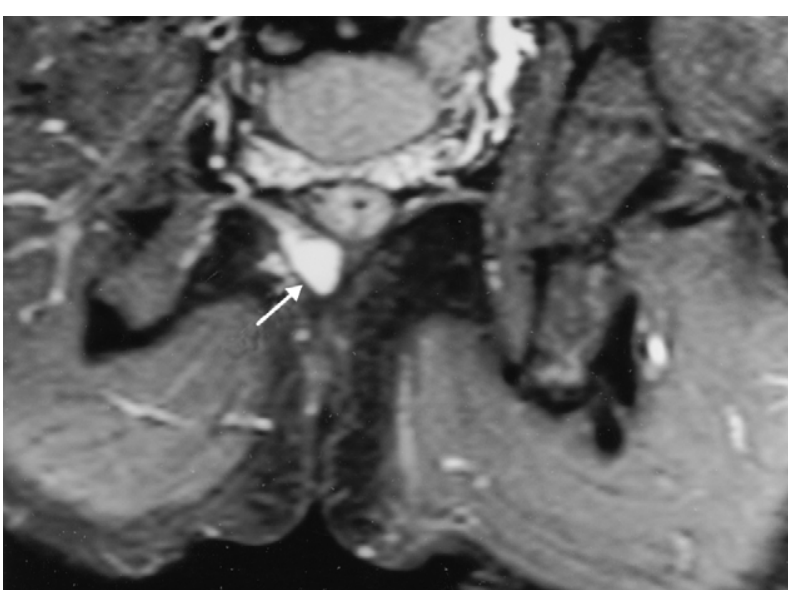

43-year-old man who had 15 previous operations for sepsis MRI shows a deep ischiorectal abscess (arrow). No external opening was present, and the operating surgeon decided not to re-explore. The patient presented again 3 months later, at which time EUA showed a collection at the site predicted by MRI.

high trans-sphincteric. Further recurrence was at the site initially predicted by MRI in all 16 patients (figure).

Overall, five $(13 \%)$ further recurrences were noted in the 40 patients in whom MRI and EUA agreed, compared with $16(52 \%)$ of the 31 in whom disagreement persisted $(p=0 \cdot 0005)$. Significantly more simple fistulas were reported in the 25 patients in whom MRI and EUA initially agreed than in those in whom the procedures disagreed $(p=0.0016)$, but no difference in complexity was reported between the other two groups.

We also investigated the relation between further recurrence and surgical attitude to discrepancy between EUA and MRI. Of the six surgeons who operated on fistulas that recurred, three always investigated discrepancy with MRI, one occasionally did, and two never did. Recurrence rate was $16 \%$, $36 \%$, and $57 \%$, respectively (four of 25 operations, nine of 25 , and eight of $14 ; \mathrm{p}=0 \cdot 008$ ).

We have shown that surgery guided by MRI can reduce further recurrence by about $75 \%$ in recurrent fistula-in-ano. Rate of further recurrence was $13.3 \%$ in fistulas in which MRI had therapeutic effect, compared with $51.6 \%$ in those in which discrepancy between imaging and EUA persisted. Moreover, further recurrence in those with disagreement was always at the site initially predicted by MRI, suggesting that surgery based on imaging would have prevented recurrence. Furthermore, surgeons who always investigated discrepancy between their own assessment and MRI had recurrence rates significantly less than those who did not.

Reasons why some surgeons choose to ignore discrepancies between MRI and EUA are complex. Self-belief varies, so that some surgeons deem re-exploration unnecessary even when confronted by a seemingly unequivocal MRI. Moreover, previous scarring hampers palpation of underlying disease, and some surgeons are not prepared to blindly incise to reach an abnormality suggested by MRI. This fact was especially true when no external opening was present. Similarly, inability to find an internal opening at EUA was strongly associated with further recurrence if MRI suggested one. MRI findings also prompted useful changes in classification, for example allowing definitive fistulotomy when supposed trans-sphincteric fistulas were revealed as intersphincteric (and vice versa), and permitting supposed supralevator extensions to be drained when imaging established them as ischiorectal. We are not suggesting that MRI can replace EUA; MRI missed some superficial extensions and had difficulty locating the precise level of the internal opening, owing to inability to visualise the dentate line.
Contributors

$S$ Halligan designed the study, wrote the protocol, obtained ethics committee approval, and maintained overall responsibility. C Bartram contributed to study design and, along with S Halligan, interpreted all MRI scans. A Williams and G Buchanan were principal investigators, and with D Tarroni, obtained data. R Phillips contributed to study design and, along with R Cohen, did most operative assessments. G Buchanan prepared the report, supervised by S Halligan. All authors approved the report.

Conflict of interest statement

None declared.

Acknowledgments

We thank all the consultant surgeons of St Mark's Hospital for allowing us to recruit themselves and their patients to this study; and Carole Jenkins and Annie Driscoll who helped to recruit patients. This study was funded by Kodak via a bursary from the Royal College of Radiologists. The sponsors had no role in study design, data collection, data analysis, data interpretation, or writing of the report.

1 Garcia-Aguilar J, Belmonte C, Wong WD, Goldberg SM, Madoff RD Anal fistula surgery: factors associated with recurrence and incontinence. Dis Colon Rectum 1996; 39: 723-29.

2 Lunniss PJ, Armstrong P, Barker PG, Reznek RH, Phillips RK. Magnetic resonance imaging of anal fistulae. Lancet 1992; 340: 394-96.

3 Spencer JA, Chapple K, Wilson D, Ward J, Windsor ACJ, Ambrose NS. Outcome after surgery for perianal fistula: predictive value of MR imaging. AfR Am F Roentgenol 1998; 171: 403-06.

4 Beets-Tan RG, Beets GL, Der Hoop AG, et al. Preoperative MR imaging of anal fistulas: does it really help the surgeon? Radiology 2001; 218: 75-84.

5 Scholefield JH, Berry DP, Armitage NCM, Wastie ML. Magnetic resonance imaging in the management of fistula in ano. Int $\mathcal{f}$ Colorect Dis 1997; 12: 276-79.

Intestinal Imaging Centre (S Halligan FRCR, Prof C Bartram FRCR), and Department of Surgery (G Buchanan fRCS, A Williams fRCS,

C R G Cohen, FRcs, D Tarroni MD, Prof R K S Phillips frcs), St Mark's Hospital, Northwick Park, London HA1 3UJ, UK

Correspondence to: Dr S Halligan

(e-mail: s.halligan@ic.ac.uk)

\section{Kidney transplantation with rabbit antithymocyte globulin induction and sirolimus monotherapy}

S John Swanson, Douglas A Hale, Roslyn B Mannon,

David E Kleiner, Linda C Cendales, Christine E Chamberlain,

Shirley M Polly, David M Harlan, Allan D Kirk

Renal allograft recipients generally need to take severa immunosuppressive agents for life. Calcineurin inhibitors and glucocorticosteroids are the mainstays of most regimens but have undesirable chronic effects. We postulated that aggressive T-cell depletion combined with the newer immunosuppressant sirolimus would permit transplantation without multidrug treatment. We therefore tested T-cell depletion with rabbit antithymocyte globulin followed by sirolimus monotherapy in $\mathbf{1 2}$ patients in an open-label study. This approach was tolerated well, and all patients achieved excellent renal function, and most did not need chronic steroid treatment or calcineurin inhibitors. Rejection was typically correlated with low concentrations of sirolimus, indicating continued dependence on maintenance immunosuppression.

Lancet 2002; 360: 1662-64

Organ transplant recipients have to take immunosuppressive drugs for life to prevent rejection of their grafts. ${ }^{1}$ Usually, such treatment includes a combination of steroids, a calcineurin inhibitor (ciclosporin or tacrolimus), and an antiproliferative agent (azathioprine or mycophenolate mofetil). Depleting or non-depleting T-cell-specific antibody preparations are also added perioperatively in many centres. Dependence on immunosuppression tempers the substantial 
benefit obtained from transplantation by increasing the risk of opportunistic infection, malignant diseases, and many non-immune side-effects. Specifically, calcineurin inhibitors are nephrotoxic, steroids exacerbate osteoporosis, and both worsen diabetes and hypertension. Methods that lessen dependence on chronic immunosuppression should thus improve life after transplantation, providing they satisfactorily prevent rejection of the grafts.

Rejection mediated by $\mathrm{T}$ cells is substantially exacerbated by a non-specific homoeostatic response to allograft ischaemia and surgical trauma. However, if $\mathrm{T}$ cells are absent during the recovery from ischaemic injury, allograft ischaemia and surgical trauma might have a reduced effect on alloimmunity, and the prevailing immune response could become one of indifference, rather than rejection. ${ }^{2}$ Furthermore, calcineurin inhibitors and steroids block function of the T-cell antigen receptor, and reliance on immunosuppression directed at this receptor can limit graft acceptance and graft rejection. ${ }^{3}$ We designed a study in which we depleted $\mathrm{T}$ cells perioperatively with polyclonal rabbit antithymocyte globulin (RATG), and added sirolimus, a drug that does not prevent $\mathrm{T}$-cell receptor signal transduction, but instead inhibits interleukin-2-receptor signalling.

Non-sensitised recipients of first, HLA-non-identical kidney transplants were offered enrolment after informed consent was obtained for participation in an Institutional Review Board approved trial. We gave recipients $2.5 \mathrm{mg} / \mathrm{kg}$ RATG (Sangstat Medical Corporation, Fremont, CA, USA) a day (over 6-12 h) for 8-10 days before transplantation (a total dose of $20 \mathrm{mg} / \mathrm{kg}$ ). The dose was reduced or stopped if the patients developed thrombocytopenia, neutropenia, or symptoms of serum sickness. We gave patients $500 \mathrm{mg}$ methylprednisolone before the first dose, and $125 \mathrm{mg}$ methylprednisolone before doses 2 and 3. We also gave patients $15 \mathrm{mg}$ sirolimus (Wyeth, Collegeville, PA, USA) on the first day after surgery, followed by $5 \mathrm{mg}$ a day thereafter. Doses were adjusted to achieve trough levels of $10-15 \mu \mathrm{g} / \mathrm{L}$. Patients received standard antimicrobial prophylaxis (ganciclovir, clotrimazole, and trimethoprimsulfamethoxazole) for 6 months or until the absolute lymphocyte count reached $0.5 \times 10^{9}$ cells $/ \mathrm{L}$. In addition to

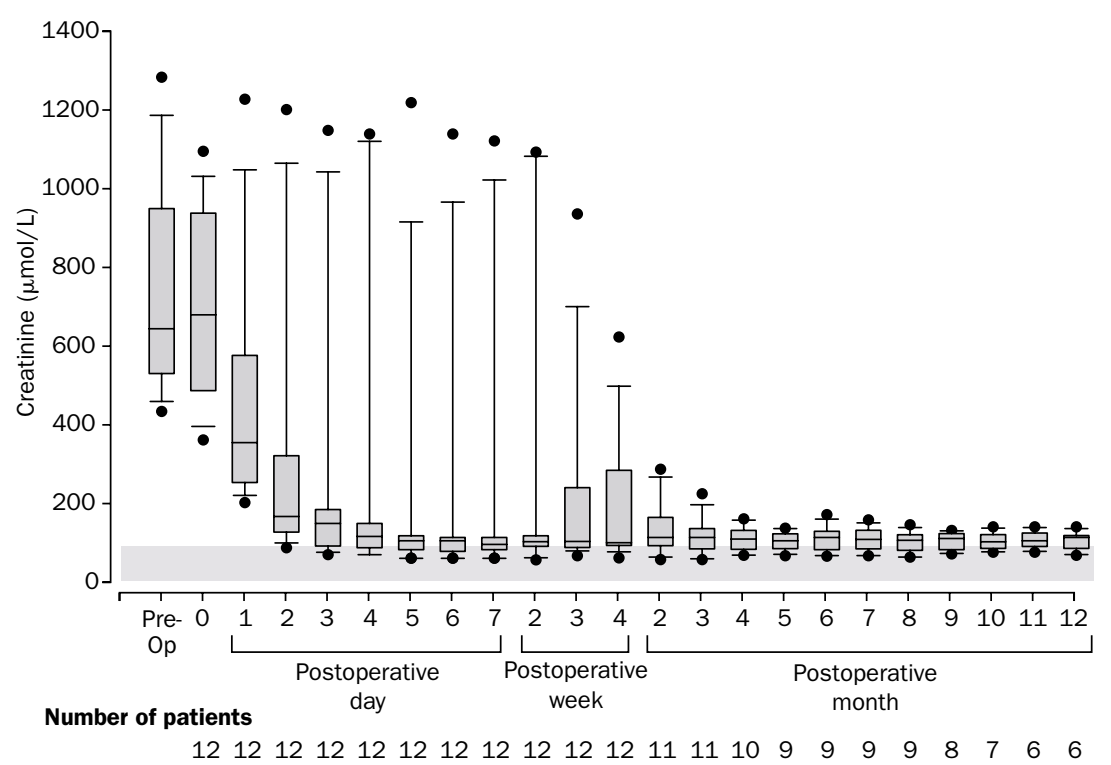

Serum creatinine in transplanted patients after RATG and sirolimus therapy

Data are mean serum creatinine for each patient in the respective time interval. The box borders are the 75 th and 25 th percentiles, and upper and lower whiskers are the 90th and 10th percentiles, respectively. Dots are the maximum and minimum values at each time point. The horizontal line is the median observation. The reference line is at $88.4 \mu \mathrm{mol} / \mathrm{L}$. standard clinical assessment, we also obtained biopsy samples from the kidney on the day of transplantation and at 1 month, 6 months, and 12 months.

We enrolled 12 patients, six of whom received cadaveric grafts, and six living donor grafts. Graft and patient survival was $100 \%$. All patients regained excellent renal function (figure). The mean creatinine concentration at 6 and 12 months was $112 \mu \mathrm{mol} / \mathrm{L}$ (SD 34) and $107 \mu \mathrm{mol} / \mathrm{L}$ (25), respectively, and the mean estimated glomerular filtration rate was $69.1 \mathrm{~mL} / \mathrm{min}(17.4)$ at 6 months and $74.2 \mathrm{~mL} / \mathrm{min}$ $(21 \cdot 3)$ at 12 months. Two patients showed signs of mild clinical rejection.

Patients received a mean of $18.6 \mathrm{mg} / \mathrm{kg}(1.95)$ of RATG over $8-10$ days (table). We had to reduce the dose in four patients. Peripheral lymphocyte depletion was excellent and sustained. Mean total lymphocyte counts were reduced by $95 \%$ on day 7 , and returned to $30 \%$ by day 180 . For 30 days after surgery, we detected no $\mathrm{T}$ cells $(\mathrm{CD} 3+)$ peripherally in any patient, after which lymphocyte counts gradually increased, but remained lower than the preoperative levels. Transient decreases in platelet and monocyte counts returned to baseline after RATG therapy.

Ten of 12 patients continued on sirolimus monotherapy. One patient was changed to tacrolimus and a reducing dose of mycophenolate mofetil at 5 months because they could not tolerate sirolimus. One patient was started on $10 \mathrm{mg}$ prednisone a day in addition to sirolimus after a rejection episode at 3 months after transplantation.

The mean dose of sirolimus needed to achieve the targeted range was $4.0 \mathrm{mg} /$ day (SD 0.6 ), although the dose needed varied greatly between patients and could not be predicted on the basis of patients' weight. Therapeutic levels were achieved through concomitant use of cytochrome P-450 inhibitors including clotrimazole and diltiazem.

Three patients developed histological features of acute cellular rejection. One episode was clinically evident at 3 months (Banff 1B) and one at 6 months (Banff 1A), and both were associated with sustained low concentrations of sirolimus $(3-5 \mu \mathrm{g} / \mathrm{L})$. Both patients responded to treatment with methylprednisolone reducing over 10 days. The patient with Banff $1 \mathrm{~B}$ continued on $10 \mathrm{mg}$ prednisone a day. The third patient showed subclinical rejection at a 6-month protocol biopsy (Banff 1A), and this rejection was also associated with a low concentration of sirolimus $(4 \cdot 2 \mu \mathrm{g} / \mathrm{L})$. We gave this patient a reducing dose of methylprednisolone over 2 weeks. In general, the histological appearance of all other protocol biopsy samples reflected the patients' excellent clinical course.

Six patients had a total of eight adverse events requiring admission, including Varicella zoster reactivation $(n=1)$ that resolved with acyclovir; bacterial urinary tract infection $(n=2)$ with infected polycystic kidneys and a pretransplantation neurogenic bladder; diabetic foot cellulitis 15 months after transplantation $(n=1)$; hernia $(n=2)$; lymphocoele needing transperitoneal drainage $(n=1)$; and postpolio motor neuropathy 1 year after transplantation $(\mathrm{n}=1)$ responsive to intravenous immunoglobulin. No patient developed lymphoproliferative disorder, malignant disease, or new fungal or viral infections after transplantation. No 


\begin{tabular}{|c|c|c|c|c|c|c|c|c|c|c|c|c|}
\hline Sex & Race & $\begin{array}{l}\text { Age } \\
\text { (years) }\end{array}$ & $\begin{array}{l}\text { Cause of } \\
\text { renal failure }\end{array}$ & Donor & $\begin{array}{l}\text { Cold } \\
\text { ischaemic } \\
\text { time (min) }\end{array}$ & A-mismatch & B-mismatch & DR-mismatch & $\begin{array}{l}\text { RATG } \\
\text { (mg/kg) }\end{array}$ & $\begin{array}{l}\text { Follow-up } \\
\text { (days) }\end{array}$ & $\begin{array}{l}\text { Most } \\
\text { recent } \\
\text { creatinine } \\
\text { mmol/L }\end{array}$ & $\begin{array}{l}\text { Glomerular } \\
\text { filtration rate } \\
(\mathrm{mL} / \mathrm{min})^{*}\end{array}$ \\
\hline M & W & 57 & Type 2 diabetes & LRD & 30 & 1 & 1 & 1 & $16 \cdot 6$ & 550 & $132 \cdot 6$ & 51 \\
\hline $\mathrm{F}$ & W & 60 & PCKD & CAD & 1740 & 1 & 2 & 2 & $15 \cdot 5$ & 529 & $70 \cdot 7$ & 78 \\
\hline$M$ & W & 26 & Reflux & LUD & 60 & 0 & 1 & 1 & $20 \cdot 2$ & 522 & $114 \cdot 9$ & 86 \\
\hline $\mathrm{M}^{*}$ & W & 44 & Medullary cystic & CAD & 1500 & 2 & 2 & 1 & $17 \cdot 3$ & 506 & $132 \cdot 6$ & 54 \\
\hline $\mathrm{F}$ & W & 31 & Lupus & CAD & 900 & 0 & 0 & 0 & $19 \cdot 4$ & 404 & $79 \cdot 6$ & 78 \\
\hline $\mathrm{M} \uparrow$ & W & 32 & GN & LRD & 90 & 1 & 1 & 1 & $17 \cdot 7$ & 403 & $106 \cdot 8$ & 75 \\
\hline$M$ & W & 54 & Type 2 diabetes & LUD & 105 & 1 & 2 & 2 & $20 \cdot 1$ & 340 & $150 \cdot 3$ & 45 \\
\hline $\mathrm{M} \dagger$ & B & 60 & GN/hypertension & CAD & 1440 & 2 & 1 & 0 & $21 \cdot 0$ & 321 & $114 \cdot 9$ & 73 \\
\hline$M$ & W & 55 & Type 1 diabetes & LRD & 45 & 1 & 1 & 1 & $19 \cdot 5$ & 298 & $106 \cdot 1$ & 67 \\
\hline$M \ddagger$ & $\mathrm{B}$ & 32 & FSGS & CAD & 900 & 2 & 2 & 2 & $19 \cdot 4$ & 165 & $221 \cdot 0$ & 46 \\
\hline $\mathrm{F} \S$ & W & 47 & Unknown & CAD & 1500 & 0 & 0 & 0 & $20 \cdot 5$ & 146 & $106 \cdot 1$ & 51 \\
\hline $\mathrm{F}$ & W & 42 & PCKD & LUD & 90 & 2 & 2 & 1 & $15 \cdot 5$ & 95 & $123 \cdot 8$ & 44 \\
\hline
\end{tabular}

$\mathrm{W}=$ white. $\mathrm{B}=$ black. $\mathrm{PCKD}=$ polycystic kidney disease. $\mathrm{LRD}=$ living related donor. $\mathrm{CAD}=$ cadaveric donor. $\mathrm{LUD}=$ living unrelated donor. $\mathrm{GN}=\mathrm{g}$ lomerulonephritis. $\mathrm{FSGS}=$ focal segmental glomerulosclerosis. *Estimated. †Clinical rejection episode. ¥Subclinical rejection episode. §Perioperative ATN.

Demographics, treatment, and outcome of all patients

patient had evidence of polyomavirus on biopsy. Side-effects associated with sirolimus included diarrhoea $(n=3)$; mouth ulcers $(n=8)$; and arthralgias $(n=2)$ that were responsive to dose reduction. Ten patients needed treatment for hyperlipidaemia.

Our patients were representative of first-time kidney recipients, including patients with delayed allograft function and poor matches. The regimen we used is critically dependent on establishment and maintenance of therapeutic concentrations of sirolimus. All rejections were associated with sirolimus concentrations below our target range, highlighting the need to monitor drug concentrations and patient compliance. Indeed, patients who were subsequently given a similar regimen without clotrimazole or diltiazem and who failed to achieve adequate concentrations of sirolimus within the first month had reversible rejection at a higher rate. We now maintain sirolimus concentrations at over $12 \mu \mathrm{g} / \mathrm{L}$.

Our dose of RATG is twice that typically used in renal transplantation. What dose of RATG is needed remains to be determined, although we would caution against equating our regimen with standard, non-investigational use of RATG. RATG has been dosed in other studies on the basis of peripheral T-cell counts. However, we believe that doses that allow detectable $\mathrm{T}$-cell counts in the periphery do not adequately deplete the secondary lymphoid tissues.

Whether other depleting agents are as effective as RATG is unclear. The choice of a polyclonal depleting agent has theoretical advantages in view of their potential for countering the effects of reperfusion injury. However, Calne and colleagues ${ }^{4}$ have shown that use of Campath- $1 \mathrm{H}$ and ciclosporin monotherapy is feasible for renal transplantation and our group and Knechtle and coworkers have initiated trials combining Campath and sirolimus with promising early results (Stuart Knechtle, personal communication).

Our results showed that monotherapy immunosuppression with sirolimus can successfully prevent acute renal allograft rejection in human beings after an appropriate induction regimen. These results have implications in investigations of allograft tolerance. Investigational immune treatments aimed at tolerance have been difficult to assess when combined with multidrug regimens, ${ }^{5}$ and there is growing concern that standard immunosuppressive regimens inhibit tolerance induction by their reliance on inhibition of $\mathrm{T}$-cell receptors. The regimen reported herein, in addition to simplifying postoperative immunosuppression, offers an alternative approach to be paired with new treatments to promote tolerance.
Contributors

S J Swanson designed and implmented the protocol, did the surgical procedure, and cared for the patients. D A Hale did the surgical procedures, cared for the patients, analysed the data, and constructed the figures. R B Mannon cared for the patients and measured and analysed renal function. D E Kleiner did the histopathological interpretation and scoring and prepared the pathology figure. L C Cendales cared for the patients and analysed the data. C C Chamberlain did the pharmaceutical care, analysed drug concentrations, and prepared the figure. S M Polly did the clinical tissue typing and analysis of antibodies. D M Harlan cared for the patients. A D Kirk designed and implemented the protocol, did the surgical procedures, and cared for the patients. All authors contibuted to the preparation of the report.

Conflict of interest statement

None declared.

\section{Acknowledgments}

We thank the nurses and transplantation coordinators of the Organ and Tissue Transplant Research Center of the Warren G Magnuson Clinical Center for their excellent patient care skills; Steven C Hoffmann, Douglas K Tadaki, and Robert L Kampen for their expert technical assistance; and Keith Hunter for his expert anaesthetic support. Sangstat Medical Corporation supplied the rabbit antithymocyte globulin for this trial. All additional material resources were supplied by the National Institute of Diabetes, Digestive and Kidney Disease. Salaries were supplied by the National Institutes of Health, and the Department of the Army as indicated by the author affiliations. The sponsors had no other role in study design, data collection, data analysis, data interpretation, or in the writing of the report.

1 Gaston RS. Maintenance immunosuppression in the renal transplant recipient: an overview. Am F Kidney Dis 2001; 38 (suppl): S25-35

2 Matzinger P. Tolerance, danger, and the extended family. Annu Rev Immunol 1994; 12: 991-1045.

$3 \mathrm{Li} \mathrm{Y,} \mathrm{Li} \mathrm{XC,} \mathrm{Zheng} \mathrm{XX,} \mathrm{et} \mathrm{al.} \mathrm{Blocking} \mathrm{both} \mathrm{signal} 1$ and signal 2 of $\mathrm{T}$-cell activation prevents apoptosis of alloreactive $\mathrm{T}$ cells and induction of peripheral allograft tolerance. Nat Med 1999; 5: 1298-302.

4 Calne R, Friend P, Moffatt S, et al. Prope tolerance, perioperative Campath-1H, and low-dose cyclosporin monotherapy in renal allograft recipients. Lancet 1998; 351: 1701-02.

5 Kirk AD, Harlan DM. Challenges for the clinical application of transplant tolerance strategies. Curr Opin Organ Transplant 2000; 5: 108-13.

Organ Transplant Service, Walter Reed Army Medical Center, Washington, DC, USA (S J Swanson MD, S M Polly MD); and Transplantation and Autoimmunity Branch, National Institute of Diabetes, Digestive and Kidney Diseases (D A Hale MD, R B Mannon MD, L C Cendales MD, D M Harlan MD, A D Kirk MD), Pharmacy Department, Clinical Center (C E Chamberlain PharmD), and Laboratory of Pathology, National Cancer Institute (D E Kleiner MD), National Institutes of Health, Bethesda, MD

Correspondence to: Dr Allan D Kirk, Room 11S/219, Building 10, Center Drive, Bethesda, MD 20892

(e-mail: allank@intra.niddk.nih.gov) 